\title{
Fusion of quantitative susceptibility maps and T1-weighted images improve brain tissue contrast in primates
}

Rakshit Dadarwal $^{*}, 1,2$, Michael Ortiz-Rios ${ }^{1,3}$, Susann Boretius ${ }^{* 1,2,3}$

1. Functional Imaging Laboratory, German Primate Center - Leibniz Institute for Primate Research, Göttingen, Germany

2. Georg-August University of Göttingen, Göttingen, Germany

3. Leibniz Science Campus Primate Cognition, Göttingen, Germany

* Corresponding authors

\section{Abstract}

Recent progress in quantitative susceptibility mapping (QSM) has enabled the accurate delineation of submillimeter scale subcortical brain structures in humans. QSM reflects the magnetic susceptibility arising from the spatial distribution of iron, myelin, and calcium in the brain. The simultaneous visualization of cortical, subcortical, and white matter structure remains, however, challenging, utilizing QSM data solely. Here we present TQ-SILiCON, a fusion method that enhances the contrast of cortical and subcortical structures and provides an excellent white matter delineation by combining QSM and conventional T1-weighted (T1w) images. In this study, we first established QSM in the macaque monkey to map iron-rich subcortical structures. Implementing the same QSM acquisition and analyses methods allowed a similar accurate delineation of subcortical structures in humans. Moreover, applying automatic brain tissue segmentation to TQ-SILiCON images of the macaque improved the classification of the brain tissue types as compared to the single T1 contrast. Furthermore, we validate our dual-contrast fusion approach in humans and similarly demonstrate improvements in automated segmentation of cortical and subcortical structures. We believe the proposed contrast will facilitate translational studies in non-human primates to investigate the pathophysiology of neurodegenerative diseases that affect the subcortical structures of the basal ganglia in humans. 


\section{Keywords}

Subcortex; Human; Macaque; Brain; T1-weighted; Quantitative Susceptibility Mapping; Segmentation

\section{Highlights}

- The subcortical gray matter areas of macaque monkeys are reliably mapped by QSM, much as they are in humans.

- Combining T1w and QSM images improves the visualization and segmentation of white matter, cortical and subcortical structures in the macaque monkey.

- The proposed dual contrast TQ-SILiCON provides a similar image quality also in humans.

- TQ-SILiCON facilitates comparative and translational neuroscience studies investigating subcortical structures. 
Dadarwal et al, 2021

\section{Introduction}

Precise delineation of brain structures plays a crucial role in neuroscience and medical imaging. Segmentation of brain structures is crucial for surgical planning and image-guided intervention and for the follow-up monitoring of brain changes in health and disease. In particular, quantitative analyses of tissue morphology and clinical diagnosis strongly rely on the precise segmentation of cortical and subcortical structures. For example, during interventional applications in primates, precise targeting of subcortical structures is critical for the delivery of pharmacological and optogenetic agents into deep brain nuclei and is aided by cortical brain tissue segmentation during MRI-guided interventions.

In both humans and non-human primates (NHPs), T1-weighted images (T1w) are widely used for the anatomical visualization of brain tissue structures. T1w provides excellent contrast for the segmentation of brain tissue classes such as gray matter, white matter, and cerebrospinal fluid. To better segment brain tissue, it is common to apply automatic volumetric and surface-based algorithms to the anatomical T1w datasets (Postelnicu et al., 2009). However, automated segmentation algorithms underperform in NHPs on T1w images, mainly due to inhomogeneities arising from the use of surface coils. Furthermore, on T1w, subcortical structures appear very similar to white matter, hampering a correct segmentation. As a result, when using $\mathrm{T} 1 \mathrm{w}$, automatic tissue segmentation algorithms may misclassify subcortical deep gray matter nuclei as part of white matter tissue in humans and NHPs.

In contrast to T1w, Quantitative Susceptibility Mapping (QSM) provides vibrant contrast in subcortical deep gray matter nuclei, particularly within basal ganglia due to the high iron abundance within these structures (Langkammer et al., 2012; Ramos et al., 2014). QSM is a rapidly evolving technique that uses gradient-recalled echo phase images to quantify the spatial distribution of magnetic susceptibility (Haacke et al., 2005, 2004). QSM contrast arises from the magnetic components in the tissue, such as iron, myelin, and calcium. The presence of myelin in the white matter results in diamagnetic susceptibility (Langkammer et al., 2012). In contrast, the presence of tissue iron in the form of ferritin macromolecules is the predominant contributor to the paramagnetic susceptibility of gray matter, including the cortex and subcortical structures. Significant progress in the post-processing of QSM data has enabled the accurate identification of small subcortical structures in humans (Guan et al., 2019; Schenck and Zimmerman, 2004). In particular, changes in the QSM maps in the basal ganglia have emerged as a potential biomarker for Parkinson's disease and other neurodegenerative diseases (Guan et al., 2019; 
Schenck and Zimmerman, 2004; Shahmaei et al., 2019). However, our current understanding of the underlying sources that might give rise to contrast changes within basal ganglia and cortex remains elusive.

In this context, comparative neuroimaging in NHPs may be of particular value. NHPs offer similar brain organization as that of humans, and established knowledge exists about the anatomical and functional organization of the cortico-striatal-thalamic circuitry, much of which had been gained from immunohistochemistry (Hadaczek et al., 2016), pharmacological (Baron et al., 2002), neurophysiological and microsimulation (Nambu et al., 2015) techniques. Despite the gain in knowledge, the translation gap between non-invasive NHP research and human neuroimaging remains large. As an example, while QSM has been widely utilized in several human neuroimaging studies (Blezer et al., 2007; Haacke et al., 2009; Langkammer et al., 2016; Ravanfar et al., 2021; Shmueli et al., 2009), QSM has not been adopted as rapidly in the NHPs neuroimaging community (Dadarwal et al., 2021, 2019; Yoshida et al., 2021).

Given the rich contrast in subcortical structures arising from high-resolution QSM and the graywhite contrasts available in T1w images, we aimed to develop a method for improving the visualization and segmentation of cortical and subcortical gray matter structures using the information provided by both T1w and QSM contrast. Toward this end, we derive a fusion image by merging T1w and QSM images using a linearly-weighted fusion algorithm which we term T1w-QSM synthetic images via a linearly-weighted combination of contrasts (TQ-SILICON).

Our method has the potential to improve the MRI-guided estimation of deep brain stimulation sites in NHPs. Additionally, we tested our approach in healthy humans, demonstrating the potential of our dual-contrast acquisition and segmentation algorithm for applications across both primate species.

\section{Materials and Methods}

\subsection{Animals and human volunteers}

In the present study, four healthy long-tailed female cynomolgus macaques (Macaca fascicularis) and three male human adults were used to obtain all neuroimaging data. The four adult macaques were between the ages of 7.7 - 8.7 years old (weighing between 3.9 and $6 \mathrm{~kg}$ ). The three human adults were between the ages of 25 and 30 years. Measurements on human 
volunteers were performed after written informed consent. The protocol was reviewed by the ethics committee of the Georg-August-University of Göttingen.

\subsection{Animal experiments}

All monkeys were purpose-bred, raised, and housed according to the standards for macaques of the German Primate Center (Göttingen, Germany). All aspects of the study were conducted in accordance with national and international guidelines of the German Animal Protection Law and the European Union Directive 2010/63/EU for the Protection of Animals used for Scientific Purposes. The study was approved by the local authorities, the Animal Welfare Service, Lower Saxony State Office for Consumer Protection and Food Safety (license-number 33.19-4250204-16/2278).

In preparation for anesthesia, the macaques were deprived of food overnight. Anesthesia was induced by a mixture of ketamine $(8.05 \pm 2.65 \mathrm{mg}$ per $\mathrm{kg}$ body weight) and medetomidine (0.02 $\pm 0.01 \mathrm{mg}$ per $\mathrm{kg})$ and maintained by isoflurane $(0.8-1.7 \%$ in oxygen and ambient air) via endotracheal tube and pressure-controlled active ventilation. The monkeys were placed in a prone position, and their heads were fixed in an MR-compatible stereotactic apparatus (Kopf $1430 \mathrm{M}$, https://kopfinstruments.com/product/model-1430m-mri-stereotaxic-instrument/).

\subsection{MRI data acquisition}

All data were acquired with a 3T MR system (MAGNETOM Prisma, Siemens Healthineers, Erlangen) equipped with a $7 \mathrm{~cm}$ single loop coil for macaque and a 20-channel head coil for human brain imaging. The imaging protocol included anatomical T1w and multi-echo gradientrecalled echo (ME-GRE) acquisitions. MR parameters for both macaques and humans are shown in Table 1.

\begin{tabular}{|c|c|c|}
\hline \multicolumn{3}{|c|}{ Macaque } \\
\hline Parameters & T1w & QSM \\
\hline Pulse sequence & 3D MPRAGE & 3D ME-GRE \\
\hline Native resolution $\left(\mathrm{mm}^{3}\right)$ & $0.5 \times 0.5 \times 0.5$ & $0.31 \times 0.31 \times 0.31$ \\
\hline Field of view $\left(\mathrm{mm}^{2}\right)$ & $108 \times 128$ & $97 \times 120$ \\
\hline Matrix size & $216 \times 256$ & $312 \times 384$ \\
\hline
\end{tabular}




\begin{tabular}{|c|c|c|}
\hline Total averages & 2 & 1 \\
\hline Total acquisition time (min) & 14.3 & 24 \\
\hline TR; TE (ms) & $2.7 / 2700$ & $57 /[3.7 / 4.9 / 48]$ \\
\hline Inversion time (ms) & 850 & -- \\
\hline Flip angle (degrees) & 8 & 20 \\
\hline Pixel Bandwidth (Hz/Px) & 250 & 250 \\
\hline \multicolumn{3}{|c|}{ Human } \\
\hline Parameters & T1w & QSM \\
\hline Pulse sequence & 3D MPRAGE & 3D ME-GRE \\
\hline Native resolution $\left(\mathrm{mm}^{3}\right)$ & $0.8 \times 0.8 \times 0.8$ & $0.75 \times 0.75 \times 0.75$ \\
\hline Field of view $\left(\mathrm{mm}^{2}\right)$ & $300 \times 320$ & $195 \times 240$ \\
\hline Matrix size & $240 \times 256$ & $260 \times 320$ \\
\hline Total averages & 1 & 1 \\
\hline Total acquisition time (min) & 6.3 & 7 \\
\hline TR; TE (ms) & $2.2 / 2400$ & $41 /[4.5 / 4.5 / 36]$ \\
\hline Inversion time (ms) & 1000 & -- \\
\hline Flip angle (degrees) & 8 & 20 \\
\hline Pixel Bandwidth (Hz/Px) & 220 & 260 \\
\hline
\end{tabular}

Table 1: Macaque and human T1w and QSM data acquisition parameters. Abbreviations: TR repetition time; TE - echo time.

\subsection{Data analyses}

T1w and ME-GRE images of both macaque and humans were converted from dicom to nifti format using the function Dcm2niix (Li et al., 2016). ME-GRE magnitude images were pixel-wise averaged across echo times. T1w and mean ME-GRE magnitude images were denoised using ANTs Denoiselmage (Avants et al., 2011). The denoised volumes were then used to manually create brain masks using the ITK-SNAP segmentation tool (Yushkevich et al., 2006). We followed a manual segmentation process due to the unavailability of sufficient automatic brain 
segmentation tools for the cynomolgus macaque brain. These brain masks were then used to correct T1w images from the bias fields using ANTs N4BiasFieldCorrection (Avants et al., 2011). The skull-stripped mean ME-GRE magnitude images were affinely registered to the subject $\mathrm{T} 1 \mathrm{w}$ brain volume using the ANTs registration algorithm.

QSM maps of monkeys and humans were reconstructed using coil combined ME-GRE phase data. This QSM reconstruction included phase unwrapping using the best-path algorithm, background field removal using Laplacian boundary value and variable spherical mean value filtering algorithms, and solving the inversion problem using multiscale dipole inversion approach (Abdul-Rahman et al., 2007; Acosta-Cabronero et al., 2018; Zhou et al., 2014).

To create symmetric brain atlases for better comparison, T1w and QSM images were duplicated and mirrored to the hemispheric plane. Using ANTs nonlinear registration (Avants et al., 2011), we constructed symmetric population-averaged T1w atlases of macaque and humans by aligning all of the native and mirrored scans. The output deformation maps from subject mean ME-GRE magnitude to symmetric population-averaged $\mathrm{T} 1 \mathrm{w}$ atlas were then used to create symmetric population-averaged QSM atlases for both macaques and humans.

A weighted linear combination was used to fuse symmetric population-averaged T1w and QSM atlases. The weights $(W)$ for T1w were generated at random between 0 and 1, while for QSM, between -1 and 0 . T1w signal intensities and QSM values $(X)$ were normalized to a range of 0 to 1 before entering it into the fusion equation,

$$
Y i=\sum_{c=0}^{c=1} \quad \text { Wic Xc }
$$

The generated synthetic images $(Y i)$ were evaluated for only unique contrasts by discarding images with poor contrast-to-noise ratio (CNR) between all tissue classes (e.g., CSF, cortical and subcortical gray matter, and white matter) and based on a linear discriminant analysis (LDA) accuracy score. The LDA model was trained with Scikit-learn (Pedregosa et al., 2011) to identify tissue classes that were manually selected on T1w and QSM atlases by drawing respective regions-of-interest (ROIs) using the ITK-SNAP tool (Yushkevich et al., 2006). These ROls were also used to calculate the CNR between gray matter and white matter, subcortical gray matter and white matter, and gray matter and subcortical gray matter. The CNRs were estimated using the following formula:

$$
C N R=\frac{\text { mean }(\text { class } X i)-\text { mean }(\text { class } X j)}{\text { standard deviation }(C S F)}
$$


Where, class Xi refers to white matter and gray matter tissue, while class Xj refers to gray matter and subcortical gray matter tissue.

In order to automatically select the most promising image fusion (i.e., weights), CNRs and LDA accuracy score were used as follow: For the average macaque brain, the minimum threshold set for the CNR between gray matter and white matter was 3, while the threshold for the CNR between subcortical gray matter and white matter was 2 . Moreover, to maintain the low contrast differences between the gray matter and subcortical gray matter, the maximum CNR threshold was set to 0.5 . The threshold for the LDA accuracy score was set to 0.90 . The selected fusion image obtained the highest LDA accuracy score out of 50 iterations and met all of the CNR requirements.

To evaluate the potential of the created fusion image for automatic tissue segmentation, ANTs Atropos was applied on both single-subject T1w images and TQ-SILiCON of macaques and humans. In the case of TQ-SILiCON, CSF was extracted beforehand, and a two-class segmentation (gray and white matter) was performed.

\section{Results}

This work aimed to establish multi-contrast imaging based on T1w and high-resolution QSM in NHPs. Below we describe our T1w and QSM results for identifying specific tissue structures in the cortex and subcortical gray matter nuclei. From the T1w and QSM images, we developed a fusion technique called TQ-SILiCON, enabling the improved automatic segmentation of white matter and cortical and subcortical gray matter. Importantly, we also evaluated our fusion strategy in humans and showed the potential of our approach for translational studies.

\subsection{Establishing QSM in NHPs}

T1w images of the macaque brain provided excellent gray-to-white matter contrast along the cortical surface. However, major subcortical deep gray matter nuclei remained challenging to delineate on T1w. In subcortical structures, T1w contrast largely delineated the caudate and putamen from the adjacent white matter, while the remaining deep gray matter nuclei - contrastwise - were identical to the neighboring white matter (Fig. 1A and B). In contrast, QSM substantially enhanced the visibility of subcortical deep gray matter nuclei from other parts of the macaque brain (Fig. 1A). Enhanced contrast on the QSM map clearly delineated subcortical structures such as caudate, putamen, external and internal segments of globus pallidus, 
thalamus, substantia nigra, and red nucleus. Due to their paramagnetic contrast, all of these deep gray matter nuclei appeared bright on the QSM map compared to the surrounding dark appearing white matter areas with diamagnetic susceptibility contrast (Fig 1).

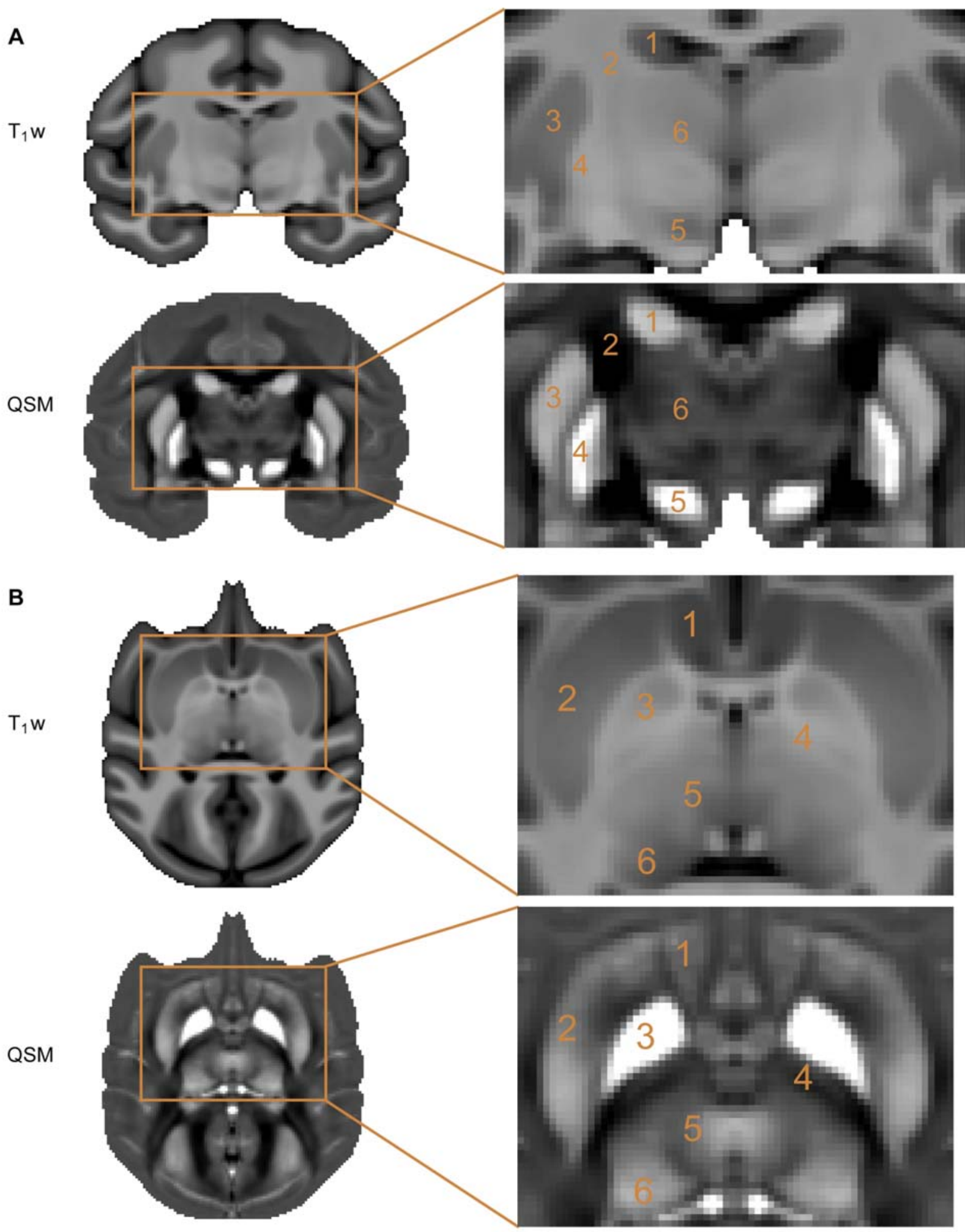


Figure 1: A. T1w and QSM coronal images of the macaque brain. T1w images provide excellent contrast between white and gray matter. However, a magnified visualization of subcortical structures reveals relatively low contrast. On the other hand, QSM coronal images provide a unique contrast in the subcortical structures but low contrast in the white and gray matter. The locations of iron-rich subcortical structures are indicated on the magnified visualization of T1w and QSM images: 1 - caudate, 2 - internal capsule, 3 - putamen, 4 - globus pallidus, 5 - substantia nigra, and 6 - thalamus. B. T1w and QSM axial images of the macaque brain. The marked areas on the magnified visualization of T1w and QSM images are 1 - accumbens, 2 - putamen, 3 - globus pallidus, 4 - internal capsule, 5 - thalamus, and 6 - pulvinar.

Interestingly, QSM contrast of the macaque thalamus highlighted different subnuclei such as pulvinar based on their varying susceptibility patterns (Fig. 1B). Among all subcortical deep gray matter nuclei in the macaque brain, the globus pallidus had the highest QSM contrast, followed by the substantia nigra, caudate, putamen, red nucleus, and thalamus, reflecting a distinct amount of iron concentration. An important feature to highlight from the QSM contrast was the clear distinction of the internal and external segments of the globus pallidus. Furthermore, QSM contrast allowed precise delineation of subcortical deep gray matter structures (Fig. 2).
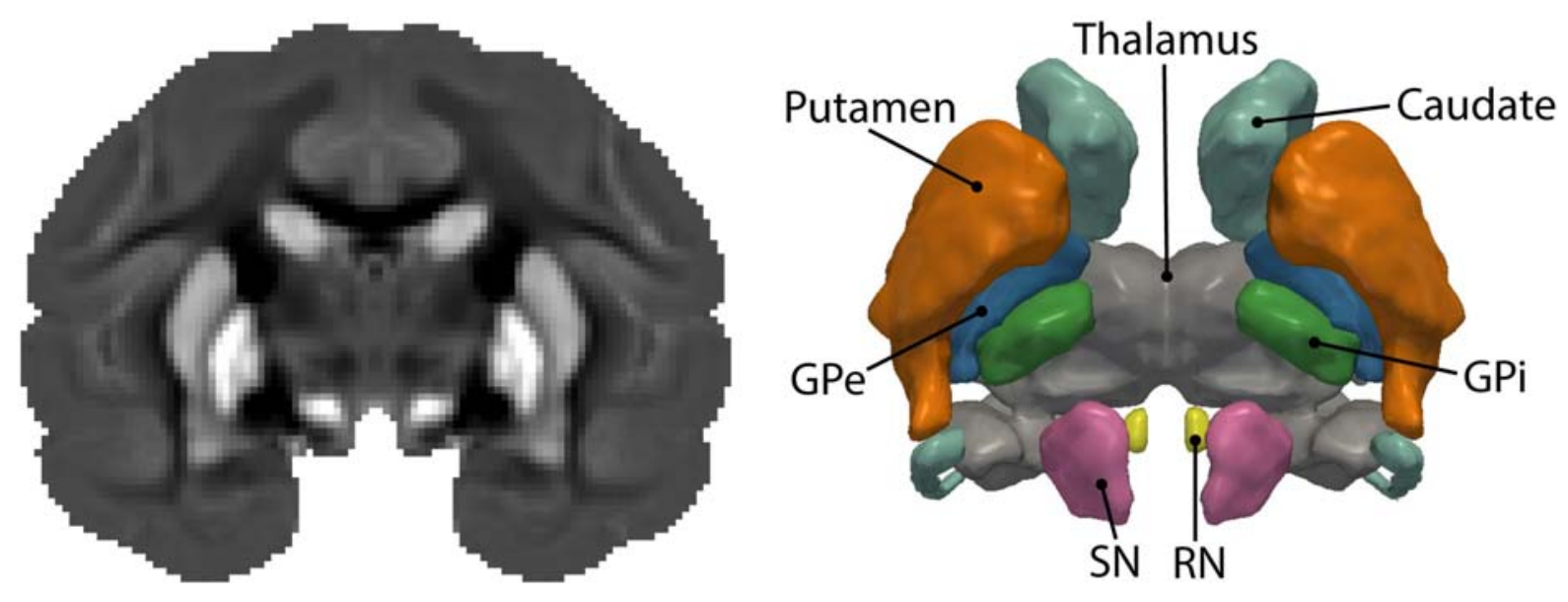

Figure 2: QSM coronal image of the macaque brain showing the iron-rich subcortical structures on a 3D rendering: putamen, caudate, globus pallidus external (GPe), globus pallidus internal (GPi), thalamus, substantia nigra $(\mathrm{SN})$, and red nucleus $(\mathrm{RN})$.

While the QSM map provided improved visualization of the iron-rich subcortical deep gray matter from the rest of the brain (Fig. 2), QSM contrast lacked the high gray-white matter contrast as compare to T1w along the cortical surface and in deep brain areas such as those of the hippocampus. To circumvent the constraint of a single contrast, we pursued an analytical strategy that combines two MRI contrasts that are sensitive to different tissue substrates. Next, 
we demonstrate the linearly-weighted combination of T1w and QSM images which provides some promising results applied to both NHPs and human neuroimaging data.

\subsection{Merging T1w and QSM provides a 3D data set with enough of contrast for segmenting subcortical and cortical tissue in NHPs}
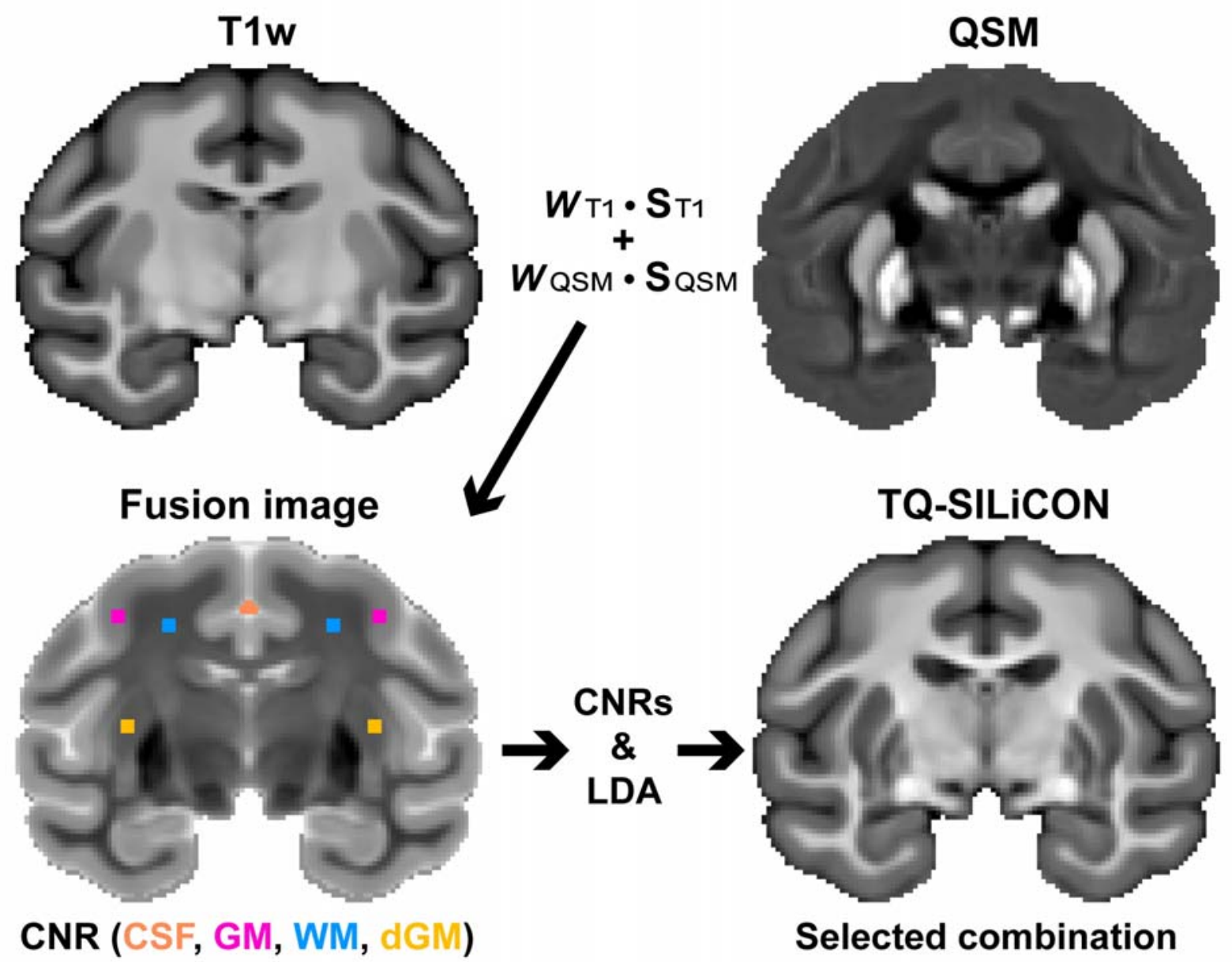

Figure 3: The TQ-SILiCON data analysis workflow. T1w and QSM images were fused using a linearlyweighted combination. WT1 and WQSM are randomly generated weights for the T1w and QSM images, while ST1 and SQSM are the normalized signal intensities of the T1w and QSM images. The generated fused images were then evaluated based on their contrast-to-noise ratio (CNR) and linear discriminant analysis (LDA) accuracy score between different tissue classes (CSF, GM, WM, and dGM). Abbreviations: CSF - cerebrospinal fluid, GM - gray matter, WM - white matter, and dGM - subcortical deep gray matter.

We devised a workflow for fusing T1w and QSM images (Fig. 3), which uses a linearly-weighted combination of the two image contrasts. The data-driven approach selected a fusion image with 
weight combinations of 0.83 and -0.87 for the T1w and QSM images, respectively. The output TQ-SILiCON image had a CNR value of 3.3 between gray matter and white matter, 2.8 between subcortical gray matter and white matter, and 0.4 between gray matter and subcortical gray matter. In addition, for the average macaque brain, TQ-SILiCON had the highest LDA accuracy score of 0.93 .

A

$\mathrm{T}_{1}$ w:TQ-SILICON

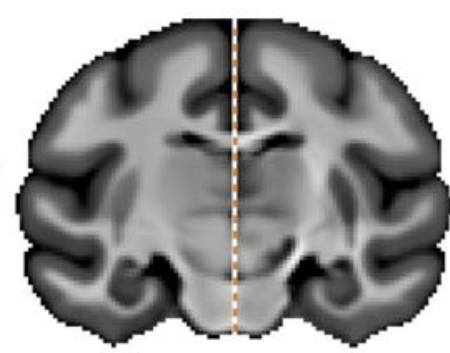

B

QSM:TQ-SILICON

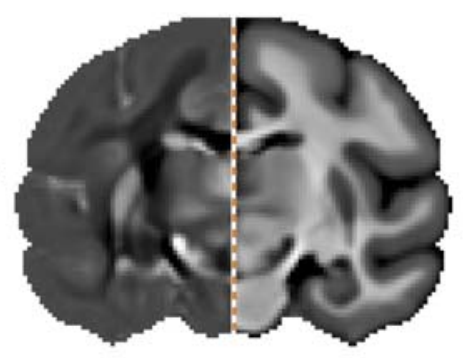

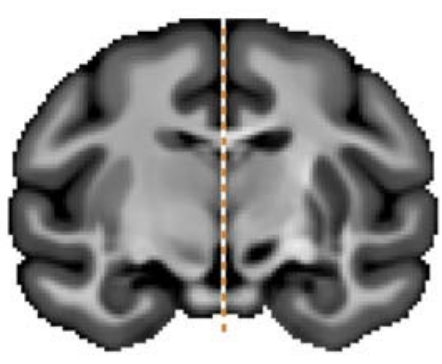
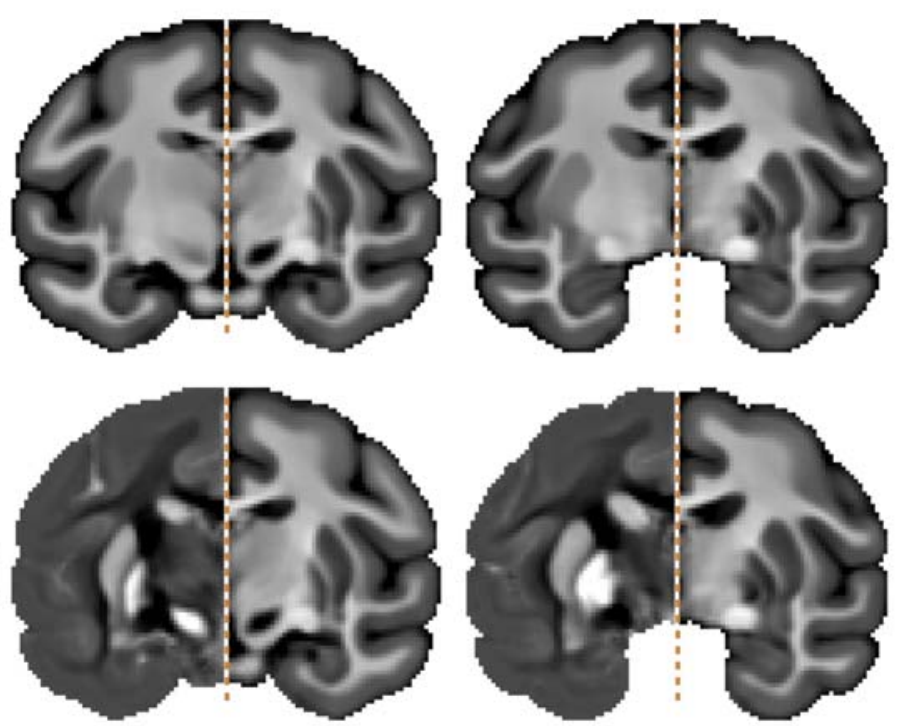

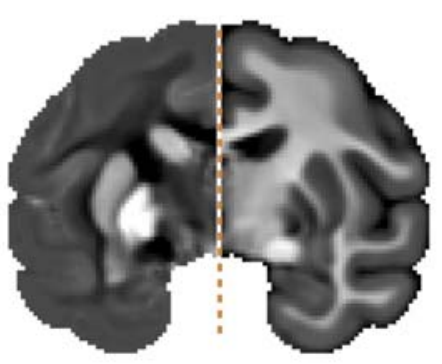

Figure 4: A. The TQ-SILiCON (right) coronal images of an average macaque template are compared to the average T1w (left). B. Similarly, the TQ-SILiCON (right) is compared to QSM images (left). In comparison to the T1w scan, the TQ-SILiCON has enhanced subcortical contrast while maintaining high gray and white matter contrast, which the QSM map lacks. 

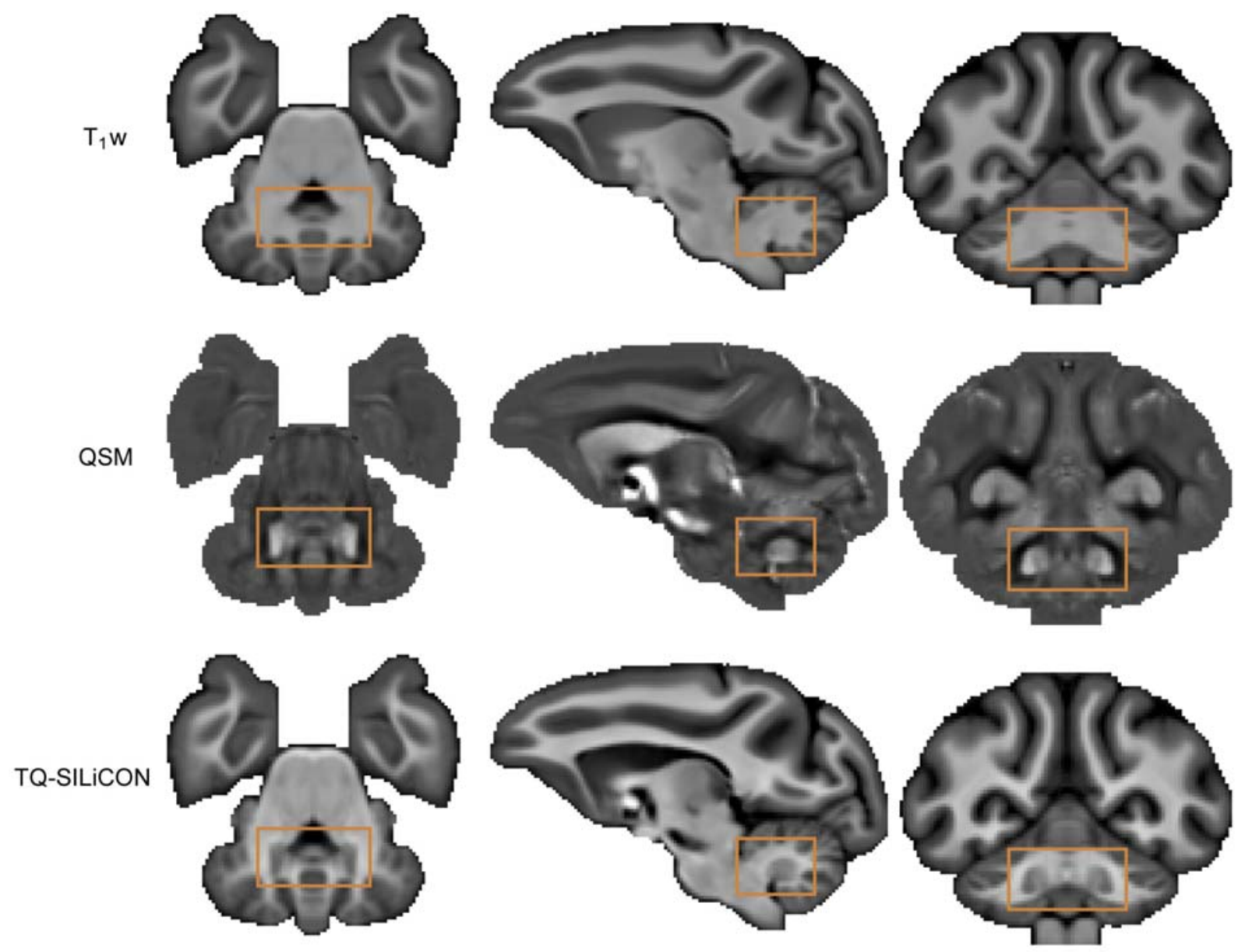

Figure 5: T1w, QSM, and TQ-SILiCON in the axial, sagittal, and coronal planes highlighting the dentate nucleus in the cerebellum of the macaque monkey. Notice the enhanced contrast (shaded orange square) of the dentate in both the QSM and the TQ-SILiCON images.

In macaques, the average TQ-SILiCON showed both excellent gray-to-white matter and deep gray matter contrast. It amplified the contrast in subcortical structures and maintained white matter landmarks in delineating subcortical structures from adjacent white matter tissue. In comparison to the average T1w image, TQ-SILiCON is superior in the delineation of subcortical structures (Fig. 4). Anatomically, we were able to identify the following nuclei (Fig. 4-6): Caudate, putamen, external and internal globus pallidus, thalamus, substantia nigra, red nucleus, and dentate nucleus. When comparing the average TQ-SILiCON image to the average QSM map, the TQ-SILiCON image showed exceptional gray-white contrast evidently not present in the QSM map (Fig. 4-6). 


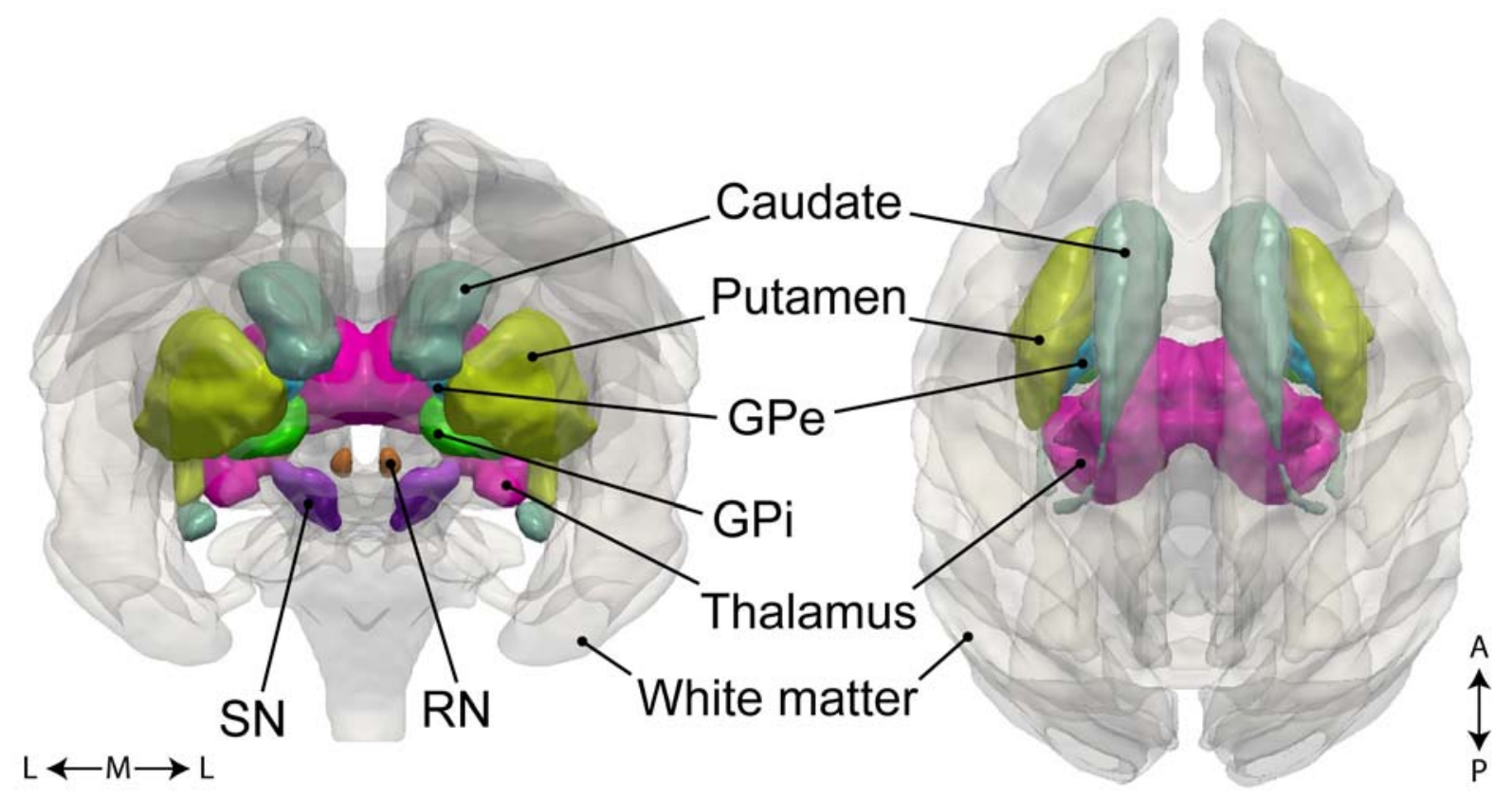

Figure 6: TQ-SILiCON allows whole-brain cortical and subcortical segmentation of the brain tissue. The white matter surface (transparent) of the macaque brain allows visualization of the color segmented subcortical deep gray matter nuclei. The highlighted subcortical nuclei include the caudate, putamen, globus pallidus external (GPe), globus pallidus internal (GPi), thalamus, substantia nigra (SN), and red nucleus (RN). Lateral (L) - Medial (M) - Lateral (L) and Anterior (A) - Posterior (P).

To further demonstrate the applicability of our fusion technique to single-subject macaque datasets, we performed tissue segmentation of gray matter and white matter on both the original T1w volume and the TQ-SILiCON volume (Fig. 7). The segmentation showed an improved gray-white matter delineation as compared to the output segmentation from the T1w volume. T1w image-based segmentation misclassified both some subcortical structures as white matter (Fig. 7) and some white matter regions as gray matter, including the extreme capsule, external capsule, and lateral medullary lamina, which were correctly classified as white matter by TQSILiCON-based segmentation (Fig. S1). 

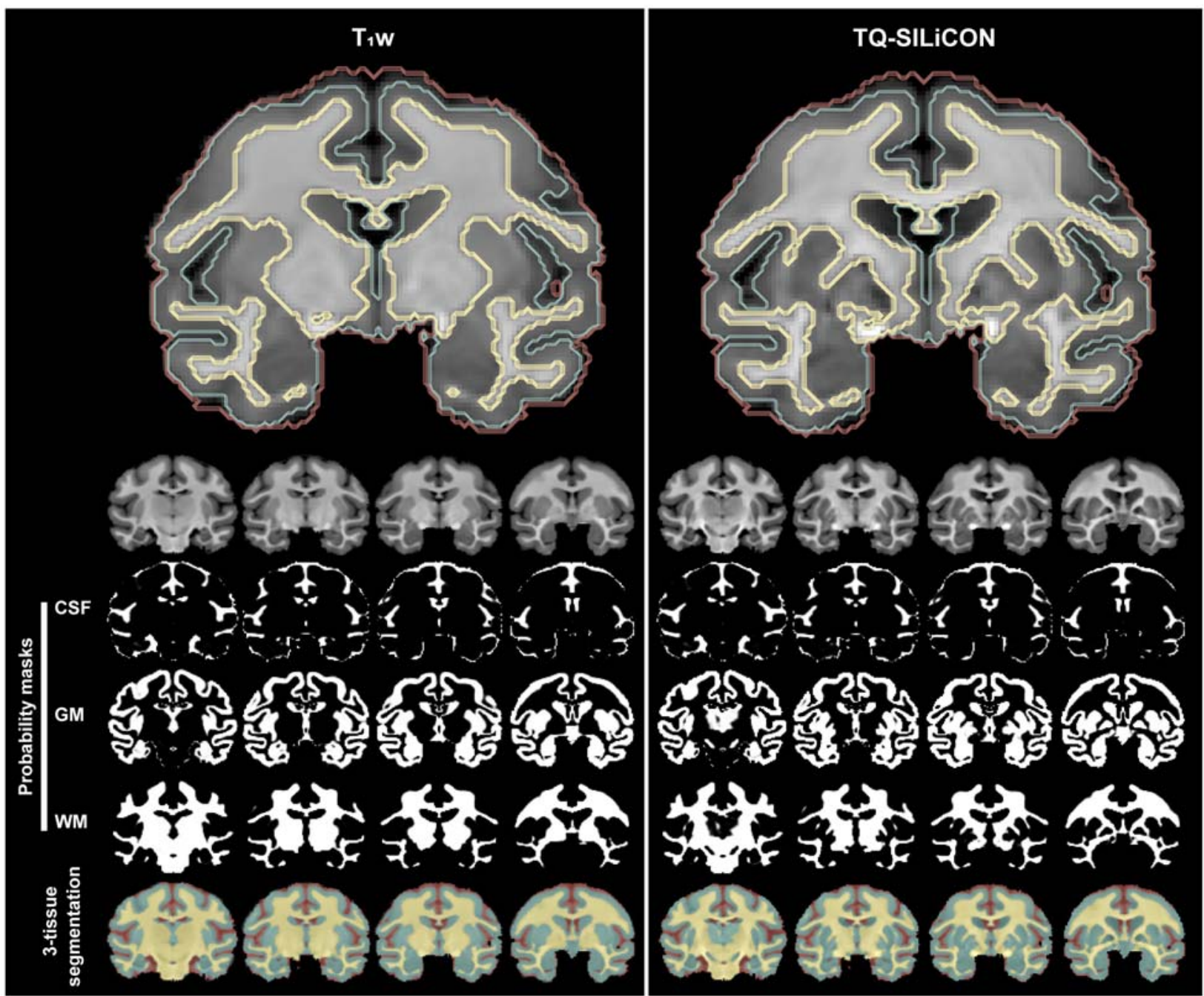

Figure 7: Coronal view of single macaque brain tissue segmentation using T1w (left) and TQ-SILiCON images (right). TQ-SILiCON enables better tissue classification of gray matter and white matter. The bottom shows the semi-transparent color-coded tissue classification of the CSF, GM, and WM. Abbreviations: CSF - cerebrospinal fluid, GM - gray matter, and WM - white matter.

In summary, our fusion technique based on T1w and QSM resulted in a contrast-enhanced T1w-like 3D dataset that improved the automated segmentation algorithm and resulted in a more precise segmentation of gray and white matter in NHPs. 


\subsection{TQ-SILiCON enables the precise segmentation of human cortical and subcortical brain tissue}

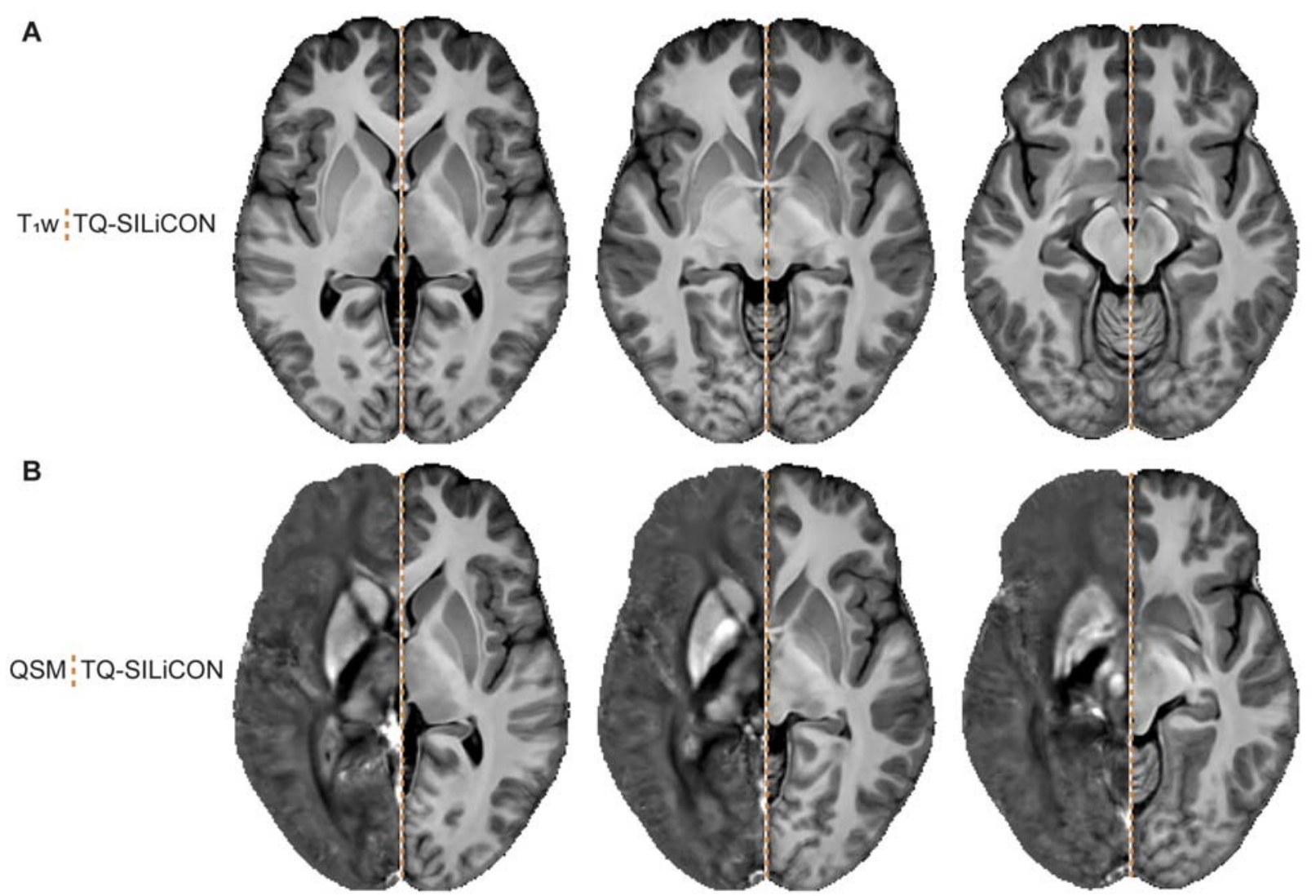

Figure 8: A. The TQ-SILiCON (right) axial images of an average human template are compared side-toside to the average T1w (left). B. Similarly, the TQ-SILiCON (right) is compared to QSM images (left). In comparison to the T1w scan, the TQ-SILiCON has enhanced subcortical contrast while maintaining high gray and white matter contrast, which the QSM map lacks.

The TQ-SILiCON method also enabled an improved tissue segmentation of the human brain. By using the same weights as in macaques (e.g., 0.83 and -0.87), TQ-SILiCON of the human brain contained both excellent gray-white and deep gray matter contrast, as illustrated in Fig. 8. In comparison to the average T1w image, the average TQ-SILICON revealed enhanced visibility and better delineation of subcortical structures. When comparing the average TQ-SILICON to the average QSM map, the TQ-SILiCON image showed exceptional T1w-like gray-white contrast that the QSM image lacked (Fig. 8). 


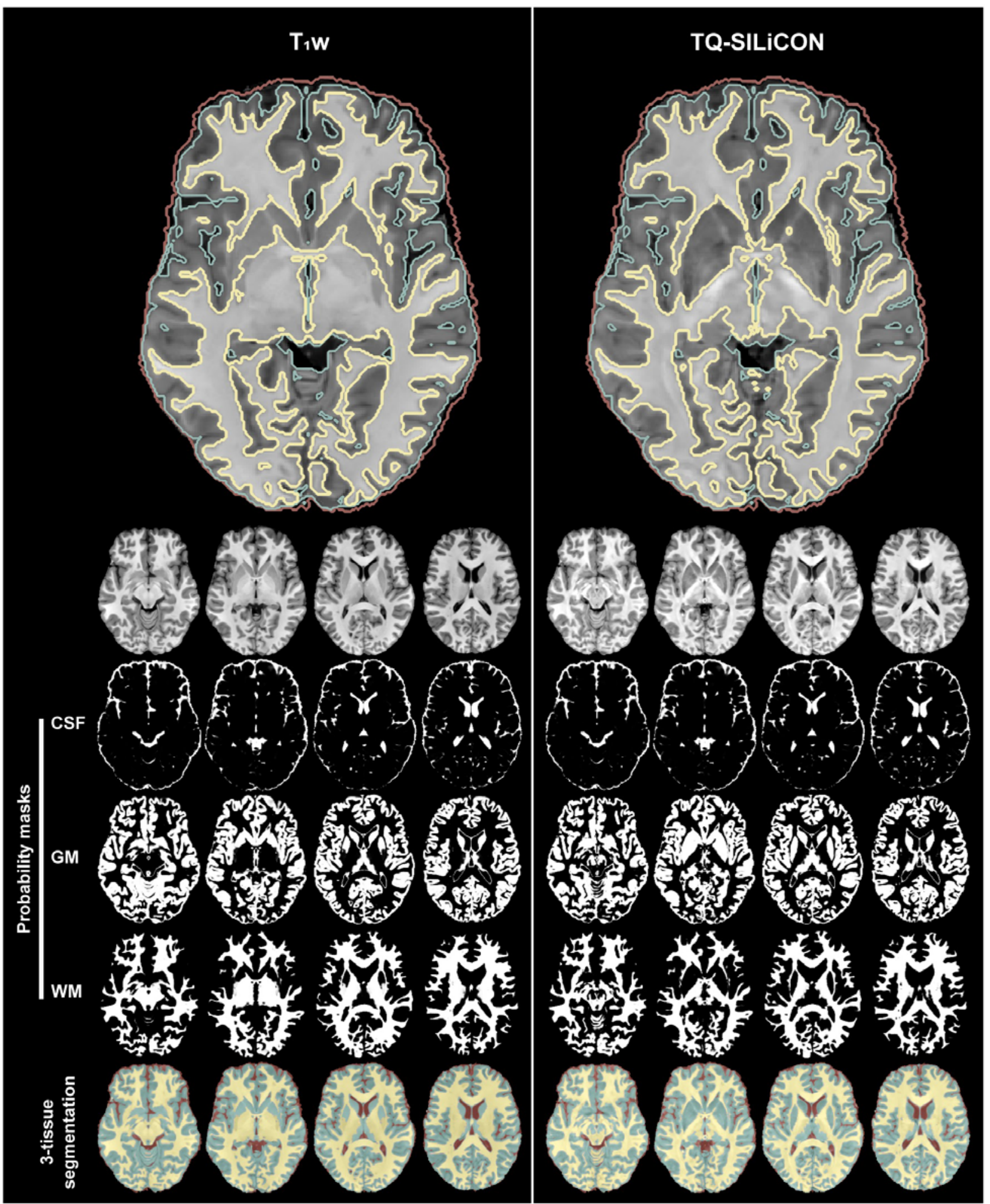

Figure 9: Axial view of single human brain tissue segmentation using T1w (left) and TQ-SILiCON images (right). TQ-SILiCON enables better tissue classification of gray matter and white matter. The bottom shows the semi-transparent color-coded tissue classification of the CSF, WM, and GM. Abbreviations: CSF - cerebrospinal fluid, GM - gray matter, and WM - white matter. 
The single-subject human segmentation results and the derived tissue probability masks are illustrated in Fig. 9. The TQ-SILiCON image-based segmentation correctly categorized the extreme capsule and external capsule as white matter and subcortical nuclei as gray matter, while the T1w image-based segmentation did not. In addition, T1w image-based segmentation misclassified white matter areas around the pre-and postcentral gyrus as gray matter in some human subjects, whereas TQ-SILiCON-based segmentation accurately classified gray matter structures from neighboring white matter areas (Fig. S2).

\section{Discussion}

In this study, we established an image contrast based on T1w and high-resolution QSM. This method, termed TQ-SILiCON, allowed us to automatically segment cortical and subcortical gray matter structures in NHPs and in humans. With the image quality achieved in a reasonable measurement time, this contrast may facilitate translational studies in NHPs for investigating the pathophysiology of subcortical structures in humans. In the following, we will first discuss the advantages of QSM, including its potential in the study of basal-ganglia circuits of primates. We will then discuss the advantages and limitations of combining QSM and T1w and how TQSILiCON could be used for neuroimaging applications in humans.

QSM is still a relatively new MRI technique that uses phase images to map the spatial distribution of paramagnetic and diamagnetic susceptibility (Haacke et al., 2005, 2004). The enhanced contrast of brain tissue largely arises from different concentrations of iron, myelin, and calcium (Haacke et al., 2005).

The absolute values derived from QSM could vary between MR-systems and measurements as they could depend on the carrier frequency of the radio pulse, the B0 field, and the frequency drift (Liu et al., 2015). However, relative magnetic susceptibilities (e.g., relative to cerebrospinal fluid or the overall brain) have been shown to be reproducible and to be a common feature, particularly of the basal ganglia in humans (Li et al., 2011; Wharton and Bowtell, 2015). As described in healthy humans and during human development, QSM values have been reproducibly linked to iron concentration (Bilgic et al., 2012). With increasing age, particularly subcortical deep gray matter structures show an increase in magnetic susceptibility in both humans (Bilgic et al., 2012; Keuken et al., 2017) and NHPs (Dadarwal et al., 2021). Moreover, increased susceptibility has been observed in neurodegenerative diseases such as amyotrophic lateral sclerosis, Alzheimer's, Parkinson's, and Huntington's disease (Haacke et al., 2005). 
In contrast to humans, only a few studies have applied QSM in NHPs (Wen et al., 2020; Yoshida et al., 2021; Dadarwal et al., 2021). The study by Yoshida et al. 2021 reported an increased magnetic susceptibility in basal ganglia structures of the rhesus macaque, in close accordance with our findings in the cynomolgus macaque. Similarly, in our QSM maps, we observed high susceptibility in the caudate, putamen, globus pallidus (internal and external segments), thalamus, substantia nigra, red nucleus, and dentate gyrus of the cerebellum (Fig. 2 and 5). Importantly, while many of the enhanced subcortical structures are known to harbor iron, it is not well understood which substances and structures are most relevant for the bulk susceptibility. For instance, the degree of contribution from myelin, especially along the white matter projections zones of the basal-ganglia cerebellar circuit, is not well understood. Identifying the sources that give rise to the increased susceptibility in vivo has significant clinical implications for neurodegenerative diseases and aging (Ravanfar et al., 2021). Further immunohistochemistry studies in NHPs might provide a better understanding of the neural substrates and molecular physiological mechanisms of the iron content and could enable means for quantifying the degree of iron contribution to the QSM map of the brain. Moreover, exploring cortical and subcortical structures by neuroimaging and interventional studies in NHPs (Klink et al., 2021) may improve our understanding of the cortico-striatal-thalamic circuits and may guide therapeutic interventions. For example, the subthalamic nucleus (STN) is a typical target for deep brain stimulation (DBS) studies; its accurate delineation might aid in the physiological characterization of neuronal responses and microstimulation parameters, as has been shown in human patients undergoing DBS treatment (Liu et al., 2015). In addition, the delineation of the internal and external globus pallidus can aid in the MRI-guided targeting of the inhibitory and excitatory output of the cortico-striatal-thalamic circuit during micro-stimulation. The identification and visualization of small subcortical structures are likewise beneficial for neuroscientists implementing optogenetic (Galvan et al., 2017; Inoue et al., 2015) or chemogenetic interventions (Mimura et al., 2021). Hence, the correct delineation of the subcortical deep gray matter nuclei, while maintaining adequate anatomical information of the whole brain, is crucial for precise interventional studies and clinical applications.

While QSM provides an outstanding contrast of deep gray nuclei, white matter structures are much better delineated on T1w. So, by linearly combining these two contrasts, we used the best of both worlds (Fig. 6). As shown in the TQ-SILiCON image, our dual-fusion approach facilitated the delineation of the macaque brain's cerebral cortex, white matter, and deep gray matter nuclei resulting in an overall T1w-like appearance. We believe TQ-SILiCON can aid stereotactic interventions of small subcortical structures such as the subthalamic nucleus, and it may be 
generally helpful for interventional studies combining neuroimaging and perturbation methods in NHPs.

Another useful application for the TQ-SILiCON is for the development of brain atlases in humans and NHPs. T1w is typically used in human and NHP brain atlases due to its excellent white and gray matter contrast. However, given that $\mathrm{T} 1 \mathrm{w}$ alone is insufficient, neuroimaging studies in humans explored the use of additional MRI contrasts such as T2, T2*, and QSM to generate contrast from subcortical deep gray matter nuclei (Alkemade et al., 2017; Bazin et al., 2020; Xiao et al., 2015). The available macaque atlases such as the D99 (Saleem and Logothetis, 2012) and the NMT (Hartig et al., 2021; Seidlitz et al., 2018) incorporate parcellations based on T1w only, in combination with the prior knowledge from post-mortem histology. In the future, we believe that adding multi-contrast acquisitions might aid in the development of more precise atlas parcellations and clearer delineation of regional boundaries, although with the limitation of an increase in acquisition time. However, our proposed TQSILiCON approach might be a more viable option for obtaining a shorter in-session acquisition. The accurate segmentation of gray and white matter tissue is not only essential for basic neuroscience applications but also for clinical diagnostics. For example, during brain development, aging, and neurological diseases specific volumetric changes might occur that might require precise segmentation and delineation of the overall structure (Boedhoe et al., 2020; Liu et al., 2015). Importantly, algorithms for automatic brain tissue segmentation typically utilize template-derived tissue probability masks (CSF, gray matter, and white matter) to initiate the segmentation process of a single subject volume. TQ-SILiCON atlases may aid in the generation of more accurate probability masks. As demonstrated by our automated tissue segmentation analyses, TQ-SILICON outperformed T1w based tissue segmentation in both macaques (Fig. 7) and humans (Fig. 9). Interestingly, TQ-SILiCON not only provided a better delineation of subcortical gray matter structures, but it also improved the assignment of white matter structures such as the extreme and external capsule and white matter areas around the pre-and postcentral gyrus (Fig. S1 and S2), even in single subjects. However, one limitation of our TQ-SILiCON approach relates to the segmentation of the CSF areas, which occasionally exhibited a comparable contrast to the basal ganglia. In our analyses, we bypassed this limitation by segmenting the CSF based on T1w and then extracting the volume from the TQSILiCON volume before applying the segmentation process. In future, the TQ-SILiCON approach could be improved by incorporating more than two contrasts to attain higher segmentation accuracy. An advantage of the TQ-SILiCON, particularly for its application in humans as opposed to the advanced deep learning based multi-contrast MRI image synthesis 
approach (Yu et al., 2020), is the simplicity of implementation, which does not require extensive computational resources. The linear combination of T1w and QSM contrasts preserves the original data features, which are retained in the TQ-SILiCON image.

In conclusion, we believe that TQ-SILiCON can significantly improve the delineation of brain structures and the accuracy of morphometric studies. T1w and QSM data sets could be obtained using clinically available 3T-MR-systems and in a reasonable short acquisition time. Importantly, the method works equally well for NHPs and humans facilitating translational studies. In the future, we will aim at studying the structural underpinnings of the MRI contrast observed in-vivo and hope to contribute to a more comprehensive understanding of the underlying pathophysiology behind neurodegenerative diseases.

\section{Supplementary Materials}


Dadarwal et al, 2021

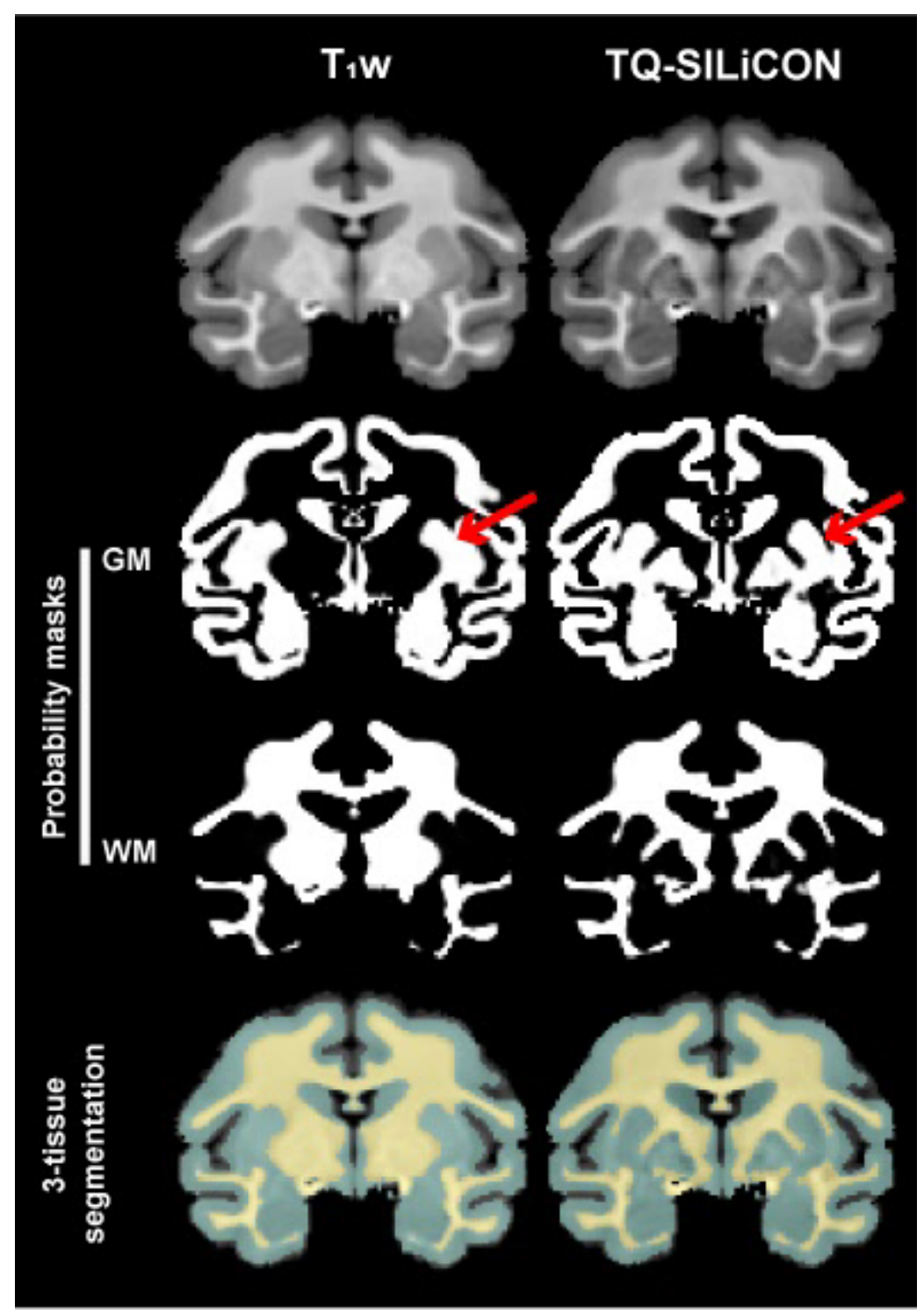

Figure S1: The coronal view of T1w and TQ-SILiCON images of a single macaque brain, along with associated GM, and WM probability masks and segmentation images. Compared to the T1W image, TQSILiCON image-based segmentation better delineates gray and white matter. Abbreviations: GM - gray matter, and WM - white matter. 


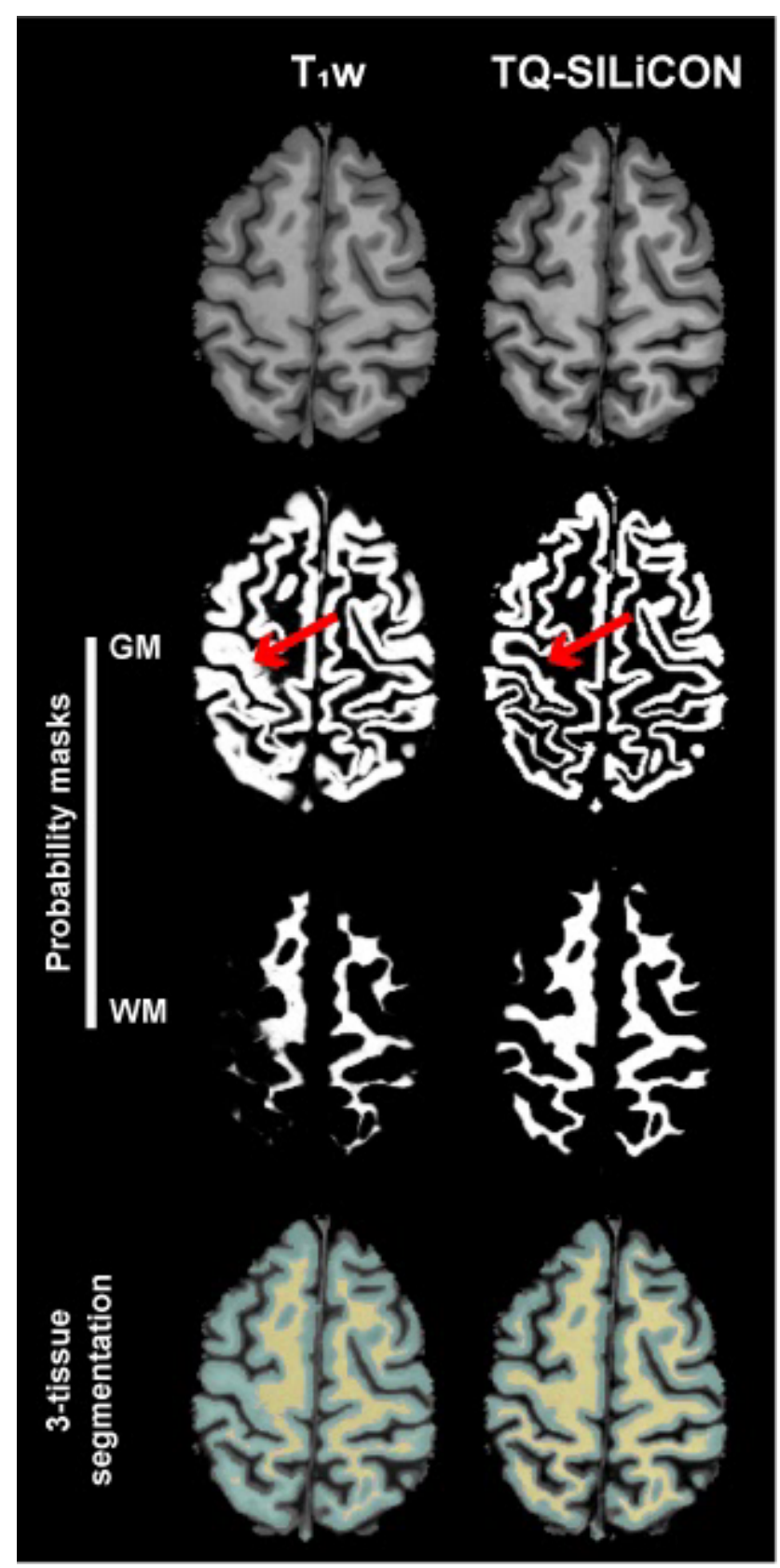

Figure S2: The axial view of T1w and TQ-SILiCON images of a single human brain, along with associated GM, and WM probability masks and segmentation images. Compared to the T1w image, TQSILiCON image-based segmentation better delineates gray and white matter. Abbreviations: GM - gray matter, and WM - white matter.

\section{References}

Abdul-Rahman, H.S., Gdeisat, M.A., Burton, D.R., Lalor, M.J., Lilley, F., Moore, C.J., 2007. Fast and robust three-dimensional best path phase unwrapping algorithm. Appl. Opt., AO 46, 6623-6635. https://doi.org/10.1364/AO.46.006623 
Acosta-Cabronero, J., Milovic, C., Mattern, H., Tejos, C., Speck, O., Callaghan, M.F., 2018. A robust multi-scale approach to quantitative susceptibility mapping. Neuroimage 183, $7-$ 24. https://doi.org/10.1016/j.neuroimage.2018.07.065

Alkemade, A., Hollander, G. de, Keuken, M.C., Schäfer, A., Ott, D.V.M., Schwarz, J., Weise, D., Kotz, S.A., Forstmann, B.U., 2017. Comparison of T2*-weighted and QSM contrasts in Parkinson's disease to visualize the STN with MRI. PLOS ONE 12, e0176130. https://doi.org/10.1371/journal.pone.0176130

Avants, B.B., Tustison, N.J., Song, G., Cook, P.A., Klein, A., Gee, J.C., 2011. A reproducible evaluation of ANTs similarity metric performance in brain image registration. Neuroimage 54, 2033-2044. https://doi.org/10.1016/j.neuroimage.2010.09.025

Baron, M.S., Wichmann, T., Ma, D., DeLong, M.R., 2002. Effects of Transient Focal Inactivation of the Basal Ganglia in Parkinsonian Primates. J. Neurosci. 22, 592-599. https://doi.org/10.1523/JNEUROSCI.22-02-00592.2002

Bazin, P.-L., Alkemade, A., Mulder, M.J., Henry, A.G., Forstmann, B.U., 2020. Multi-contrast anatomical subcortical structures parcellation. eLife 9, e59430. https://doi.org/10.7554/eLife.59430

Bilgic, B., Pfefferbaum, A., Rohlfing, T., Sullivan, E.V., Adalsteinsson, E., 2012. MRI estimates of brain iron concentration in normal aging using quantitative susceptibility mapping. Neurolmage 59, 2625-2635. https://doi.org/10.1016/j.neuroimage.2011.08.077

Blezer, E.L.A., Bauer, J., Brok, H.P.M., Nicolay, K., Hart, B.A. 't, 2007. Quantitative MRIpathology correlations of brain white matter lesions developing in a non-human primate model of multiple sclerosis. NMR in Biomedicine 20, 90-103. https://doi.org/10.1002/nbm.1085

Boedhoe, P.S.W., van Rooij, D., Hoogman, M., Twisk, J.W.R., Schmaal, L., Abe, Y., Alonso, P., Ameis, S.H., Anikin, A., Anticevic, A., Arango, C., Arnold, P.D., Asherson, P., Assogna, F., Auzias, G., Banaschewski, T., Baranov, A., Batistuzzo, M.C., Baumeister, S., BaurStreubel, R., Behrmann, M., Bellgrove, M.A., Benedetti, F., Beucke, J.C., Biederman, J., Bollettini, I., Bose, A., Bralten, J., Bramati, I.E., Brandeis, D., Brem, S., Brennan, B.P., Busatto, G.F., Calderoni, S., Calvo, A., Calvo, R., Castellanos, F.X., Cercignani, M., Chaim-Avancini, T.M., Chantiluke, K.C., Cheng, Y., Cho, K.I.K., Christakou, A., Coghill, D., Conzelmann, A., Cubillo, A.I., Dale, A.M., Dallaspezia, S., Daly, E., Denys, D., Deruelle, C., Di Martino, A., Dinstein, I., Doyle, A.E., Durston, S., Earl, E.A., Ecker, C., Ehrlich, S., Ely, B.A., Epstein, J.N., Ethofer, T., Fair, D.A., Fallgatter, A.J., Faraone, S.V., Fedor, J., Feng, X., Feusner, J.D., Fitzgerald, J., Fitzgerald, K.D., Fouche, J.-P., Freitag, C.M., Fridgeirsson, E.A., Frodl, T., Gabel, M.C., Gallagher, L., Gogberashvili, T., Gori, I., Gruner, P., Gürsel, D.A., Haar, S., Haavik, J., Hall, G.B., Harrison, N.A., Hartman, C.A., Heslenfeld, D.J., Hirano, Y., Hoekstra, P.J., Hoexter, M.Q., Hohmann, S., Høvik, M.F., Hu, H., Huyser, C., Jahanshad, N., Jalbrzikowski, M., James, A., Janssen, J., JaspersFayer, F., Jernigan, T.L., Kapilushniy, D., Kardatzki, B., Karkashadze, G., Kathmann, N., Kaufmann, C., Kelly, C., Khadka, S., King, J.A., Koch, K., Kohls, G., Konrad, K., Kuno, M., Kuntsi, J., Kvale, G., Kwon, J.S., Lázaro, L., Lera-Miguel, S., Lesch, K.-P., Hoekstra, L., Liu, Y., Lochner, C., Louza, M.R., Luna, B., Lundervold, A.J., Malpas, C.B., Marques, P., Marsh, R., Martínez-Zalacaín, I., Mataix-Cols, D., Mattos, P., McCarthy, H., McGrath, J., Mehta, M.A., Menchón, J.M., Mennes, M., Martinho, M.M., Moreira, P.S., Morer, A., Morgado, P., Muratori, F., Murphy, C.M., Murphy, D.G.M., Nakagawa, A., Nakamae, T., Nakao, T., Namazova-Baranova, L., Narayanaswamy, J.C., Nicolau, R., Nigg, J.T., Novotny, S.E., Nurmi, E.L., Weiss, E.O., O'Gorman Tuura, R.L., O'Hearn, K., O’Neill, J., Oosterlaan, J., Oranje, B., Paloyelis, Y., Parellada, M., Pauli, P., Perriello, C., Piacentini, J., Piras, Fabrizio, Piras, Federica, Plessen, K.J., Puig, O., Ramos-Quiroga, J.A., Reddy, Y.C.J., Reif, A., Reneman, L., Retico, A., Rosa, P.G.P., Rubia, K., Rus, O.G., Sakai, Y., Schrantee, A., Schwarz, L., Schweren, L.J.S., Seitz, J., Shaw, P., Shook, D., Silk, T.J., 
Simpson, H.B., Skokauskas, N., Soliva Vila, J.C., Solovieva, A., Soreni, N., SorianoMas, C., Spalletta, G., Stern, E.R., Stevens, M.C., Stewart, S.E., Sudre, G., Szeszko, P.R., Tamm, L., Taylor, M.J., Tolin, D.F., Tosetti, M., Tovar-Moll, F., Tsuchiyagaito, A., van Erp, T.G.M., van Wingen, G.A., Vance, A., Venkatasubramanian, G., Vilarroya, O., Vives-Gilabert, Y., von Polier, G.G., Walitza, S., Wallace, G.L., Wang, Z., Wolfers, T., Yoncheva, Y.N., Yun, J.-Y., Zanetti, M.V., Zhou, F., Ziegler, G.C., Zierhut, K.C., Zwiers, M.P., Thompson, P.M., Stein, D.J., Buitelaar, J., Franke, B., van den Heuvel, O.A., 2020. Subcortical Brain Volume, Regional Cortical Thickness, and Cortical Surface Area Across Disorders: Findings From the ENIGMA ADHD, ASD, and OCD Working Groups. AJP 177, 834-843. https://doi.org/10.1176/appi.ajp.2020.19030331

Dadarwal, R., Hintz, L., Moussavi, A., Boretius, S., 2019. Quantitative Susceptibility Mapping of the Brain - A Comparative In vivo Study of Humans and Nonhuman Primates [WWW Document]. URL https://scholar.google.de/citations?view_op=view_citation\&hl=en\&user=1jnx4GYAAAAJ \&citation_for_view=1jnx4GYAAAAJ:IjCSPB-OGe-̄C (accessed 9.22.21).

Dadarwal, R., Mylius, J., Boretius, S., 2021. Brain aging in cynomolgus macaques and common marmosets explored by mapping the magnetic susceptibility and $\mathrm{R}^{*}$ [WWW Document]. URL https://index.mirasmart.com/ISMRM2021/PDFfiles/2560.html (accessed 7.13.21).

Galvan, A., Stauffer, W.R., Acker, L., El-Shamayleh, Y., Inoue, K., Ohayon, S., Schmid, M.C., 2017. Nonhuman Primate Optogenetics: Recent Advances and Future Directions. J. Neurosci. 37, 10894-10903. https://doi.org/10.1523/JNEUROSCI.1839-17.2017

Guan, X., Huang, P., Zeng, Q., Liu, C., Wei, H., Xuan, M., Gu, Q., Xu, X., Wang, N., Yu, X., Luo, X., Zhang, M., 2019. Quantitative susceptibility mapping as a biomarker for evaluating white matter alterations in Parkinson's disease. Brain Imaging Behav 13, 220-231. https://doi.org/10.1007/s11682-018-9842-z

Haacke, E.M., Cheng, N.Y.C., House, M.J., Liu, Q., Neelavalli, J., Ogg, R.J., Khan, A., Ayaz, M., Kirsch, W., Obenaus, A., 2005. Imaging iron stores in the brain using magnetic resonance imaging. Magnetic Resonance Imaging 23, 1-25. https://doi.org/10.1016/j.mri.2004.10.001

Haacke, E.M., Mittal, S., Wu, Z., Neelavalli, J., Cheng, Y.-C.N., 2009. Susceptibility-Weighted Imaging: Technical Aspects and Clinical Applications, Part 1. AJNR Am J Neuroradiol 30, 19-30. https://doi.org/10.3174/ajnr.A1400

Haacke, E.M., Xu, Y., Cheng, Y.-C.N., Reichenbach, J.R., 2004. Susceptibility weighted imaging (SWI). Magnetic Resonance in Medicine 52, 612-618. https://doi.org/10.1002/mrm.20198

Hadaczek, P., Stanek, L., Ciesielska, A., Sudhakar, V., Samaranch, L., Pivirotto, P., Bringas, J., O’Riordan, C., Mastis, B., San Sebastian, W., Forsayeth, J., Cheng, S.H., Bankiewicz, K.S., Shihabuddin, L.S., 2016. Widespread AAV1- and AAV2-mediated transgene expression in the nonhuman primate brain: implications for Huntington's disease. Mol Ther Methods Clin Dev 3, 16037. https://doi.org/10.1038/mtm.2016.37

Hartig, R., Glen, D., Jung, B., Logothetis, N.K., Paxinos, G., Garza-Villarreal, E.A., Messinger, A., Evrard, H.C., 2021. The Subcortical Atlas of the Rhesus Macaque (SARM) for neuroimaging. Neurolmage 235, 117996. https://doi.org/10.1016/j.neuroimage.2021.117996

Inoue, K., Takada, M., Matsumoto, M., 2015. Neuronal and behavioural modulations by pathway-selective optogenetic stimulation of the primate oculomotor system. Nat Commun 6, 8378. https://doi.org/10.1038/ncomms9378

Keuken, M.C., Bazin, P.-L., Backhouse, K., Beekhuizen, S., Himmer, L., Kandola, A., Lafeber, J.J., Prochazkova, L., Trutti, A., Schäfer, A., Turner, R., Forstmann, B.U., 2017. Effects of aging on $\$ \$ T$ T 11$\} \$ \$, \$ T \_\{2\}^{\wedge}\left\{{ }^{*}\right\} \$$, and $Q S M$ MRI values in the subcortex. Brain Struct Funct 222, 2487-2505. https://doi.org/10.1007/s00429-016-1352-4 
Klink, P.C., Aubry, J.-F., Ferrera, V.P., Fox, A.S., Froudist-Walsh, S., Jarraya, B., Konofagou, E.E., Krauzlis, R.J., Messinger, A., Mitchell, A.S., Ortiz-Rios, M., Oya, H., Roberts, A.C., Roe, A.W., Rushworth, M.F.S., Sallet, J., Schmid, M.C., Schroeder, C.E., Tasserie, J., Tsao, D.Y., Uhrig, L., Vanduffel, W., Wilke, M., Kagan, I., Petkov, C.I., 2021. Combining brain perturbation and neuroimaging in non-human primates. Neurolmage 235, 118017. https://doi.org/10.1016/j.neuroimage.2021.118017

Langkammer, C., Pirpamer, L., Seiler, S., Deistung, A., Schweser, F., Franthal, S., Homayoon, N., Katschnig-Winter, P., Koegl-Wallner, M., Pendl, T., Stoegerer, E.M., Wenzel, K., Fazekas, F., Ropele, S., Reichenbach, J.R., Schmidt, R., Schwingenschuh, P., 2016. Quantitative Susceptibility Mapping in Parkinson's Disease. PLOS ONE 11, e0162460. https://doi.org/10.1371/journal.pone.0162460

Langkammer, C., Schweser, F., Krebs, N., Deistung, A., Goessler, W., Scheurer, E., Sommer, K., Reishofer, G., Yen, K., Fazekas, F., Ropele, S., Reichenbach, J.R., 2012. Quantitative susceptibility mapping (QSM) as a means to measure brain iron? A post mortem validation study. Neurolmage 62, 1593-1599. https://doi.org/10.1016/j.neuroimage.2012.05.049

Li, W., Wu, B., Liu, C., 2011. Quantitative susceptibility mapping of human brain reflects spatial variation in tissue composition. Neurolmage 55, 1645-1656. https://doi.org/10.1016/j.neuroimage.2010.11.088

Li, X., Morgan, P.S., Ashburner, J., Smith, J., Rorden, C., 2016. The first step for neuroimaging data analysis: DICOM to NIfTI conversion. Journal of Neuroscience Methods 264, 4756. https://doi.org/10.1016/j.jneumeth.2016.03.001

Liu, C., Li, W., Tong, K.A., Yeom, K.W., Kuzminski, S., 2015. Susceptibility-weighted imaging and quantitative susceptibility mapping in the brain. J Magn Reson Imaging 42, 23-41. https://doi.org/10.1002/jmri.24768

Mimura, K., Nagai, Y., Inoue, K., Matsumoto, J., Hori, Y., Sato, C., Kimura, K., Okauchi, T., Hirabayashi, T., Nishijo, H., Yahata, N., Takada, M., Suhara, T., Higuchi, M., Minamimoto, T., 2021. Chemogenetic activation of nigrostriatal dopamine neurons in freely moving common marmosets. iScience 24, 103066. https://doi.org/10.1016/j.isci.2021.103066

Nambu, A., Tachibana, Y., Chiken, S., 2015. Cause of parkinsonian symptoms: Firing rate, firing pattern or dynamic activity changes? Basal Ganglia 5, 1-6. https://doi.org/10.1016/j.baga.2014.11.001

Pedregosa, F., Varoquaux, G., Gramfort, A., Michel, V., Thirion, B., Grisel, O., Blondel, M., Prettenhofer, P., Weiss, R., Dubourg, V., Vanderplas, J., Passos, A., Cournapeau, D., Brucher, M., Perrot, M., Duchesnay, É., 2011. Scikit-learn: Machine Learning in Python. Journal of Machine Learning Research 12, 2825-2830.

Postelnicu, G., Zöllei, L., Fischl, B., 2009. Combined Volumetric and Surface Registration. IEEE Trans Med Imaging 28, 508-522. https://doi.org/10.1109/TMI.2008.2004426

Ramos, P., Santos, A., Pinto, N.R., Mendes, R., Magalhães, T., Almeida, A., 2014. Iron levels in the human brain: A post-mortem study of anatomical region differences and age-related changes. Journal of Trace Elements in Medicine and Biology 28, 13-17. https://doi.org/10.1016/j.jtemb.2013.08.001

Ravanfar, P., Loi, S.M., Syeda, W.T., Van Rheenen, T.E., Bush, A.I., Desmond, P., Cropley, V.L., Lane, D.J.R., Opazo, C.M., Moffat, B.A., Velakoulis, D., Pantelis, C., 2021. Systematic Review: Quantitative Susceptibility Mapping (QSM) of Brain Iron Profile in Neurodegenerative Diseases. Front Neurosci 15, 618435. https://doi.org/10.3389/fnins.2021.618435

Saleem, K.S., Logothetis, N.K., 2012. A Combined MRI and Histology Atlas of the Rhesus Monkey Brain in Stereotaxic Coordinates. Academic Press.

Schenck, J.F., Zimmerman, E.A., 2004. High-field magnetic resonance imaging of brain iron: 
birth of a biomarker? NMR Biomed 17, 433-445. https://doi.org/10.1002/nbm.922

Seidlitz, J., Sponheim, C., Glen, D., Ye, F.Q., Saleem, K.S., Leopold, D.A., Ungerleider, L., Messinger, A., 2018. A population MRI brain template and analysis tools for the macaque. Neuroimage 170, 121-131. https://doi.org/10.1016/j.neuroimage.2017.04.063

Shahmaei, V., Faeghi, F., Mohammadbeigi, A., Hashemi, H., Ashrafi, F., 2019. Evaluation of iron deposition in brain basal ganglia of patients with Parkinson's disease using quantitative susceptibility mapping. Eur J Radiol Open 6, 169-174. https://doi.org/10.1016/j.ejro.2019.04.005

Shmueli, K., Zwart, J.A. de, Gelderen, P. van, Li, T.-Q., Dodd, S.J., Duyn, J.H., 2009. Magnetic susceptibility mapping of brain tissue in vivo using MRI phase data. Magnetic Resonance in Medicine 62, 1510-1522. https://doi.org/10.1002/mrm.22135

Wen, Q., Yang, H., Li, J., Zhang, J., Tong, H., Ye, Q., Zhong, K., 2020. Ultra-High-Resolution in vitro MRI Study of Age-Related Brain Subcortical Susceptibility Alteration in Rhesus Monkeys at 9.4 T. Frontiers in Aging Neuroscience 12, 259. https://doi.org/10.3389/fnagi.2020.00259

Wharton, S., Bowtell, R., 2015. Effects of white matter microstructure on phase and susceptibility maps. Magnetic Resonance in Medicine 73, 1258-1269. https://doi.org/10.1002/mrm.25189

Xiao, Y., Fonov, V., Bériault, S., Subaie, F.A., Chakravarty, M.M., Sadikot, A.F., Pike, G.B., Collins, D.L., 2015. Multi-contrast unbiased MRI atlas of a Parkinson's disease population. Int J CARS 10, 329-341. https://doi.org/10.1007/s11548-014-1068-y

Yoshida, A., Ye, F.Q., Yu, D.K., Leopold, D.A., Hikosaka, O., 2021. Visualization of iron-rich subcortical structures in non-human primates in vivo by quantitative susceptibility mapping at 3T MRI. Neurolmage 241, 118429. https://doi.org/10.1016/j.neuroimage.2021.118429

Yu, B., Wang, Y., Wang, L., Shen, D., Zhou, L., 2020. Medical Image Synthesis via Deep Learning. Adv Exp Med Biol 1213, 23-44. https://doi.org/10.1007/978-3-030-33128-3_2

Yushkevich, P.A., Piven, J., Hazlett, H.C., Smith, R.G., Ho, S., Gee, J.C., Gerig, G., 2006. Userguided $3 \mathrm{D}$ active contour segmentation of anatomical structures: significantly improved efficiency and reliability. Neuroimage 31, 1116-1128. https://doi.org/10.1016/j.neuroimage.2006.01.015

Zhou, D., Liu, T., Spincemaille, P., Wang, Y., 2014. Background field removal by solving the Laplacian boundary value problem. NMR in Biomedicine 27, 312-319. https://doi.org/10.1002/nbm.3064 